

\title{
A INFLUÊNCIA DO AMBIENTE GEOGRÁFICO NO DESEMPENHO DOS BANCOS ISLÂMICOS
}

\author{
THE INFLUENCE OF THE GEOGRAPHICAL ENVIRONMENT ON ISLAMIC \\ BANKING PERFORMANCE
}

\author{
AHMED SAMEER EL KHATIB \\ Centro Universitário FECAP \\ Endereço: Rua da Liberdade, 532 | Liberdade | 01502-001 | \\ São Paulo, SP | Brasil. \\ (1) https://orcid.org/0000-0002-0764-8622 \\ ahmed.khatib@fecap.br
}

\section{RESUMO}

O objetivo do presente artigo é examinar a influência do ambiente geográfico no desempenho de bancos islâmicos presentes em quatro regiões: África, Ásia, Europa e América do Norte. Para atingir esse objetivo, foram utilizados dados diários de 113 bancos islâmicos entre os anos de 2010 e 2019. Foram aplicadas diferentes abordagens metodológicas, como análise de componentes principais e regressão quantílica com efeitos fixos para dados em painel. Como resultados, descobrimos que a análise dos componentes principais mostra que o desempenho dos bancos islâmicos varia entre as regiões. A regressão do painel linear destaca que o ambiente geográfico afeta positiva e significativamente o sistema bancário islâmico, sugerindo a importância dos efeitos dessa externalidade. Finalmente, o efeito ambiental parece variar com os quantis, trazendo efeito positivo para o quantil mais baixo e efeito negativo para o quantil mais alto. Essa especificação quantílica aponta para a não linearidade na relação ambientedesempenho do banco islâmico, refletindo uma disciplina variável no tempo imposta pelo conselho da Shariah ou de Lei Islâmica. Essa descoberta ajuda a explicar melhor a principal diferença entre os bancos islâmicos no oriente e os do ocidente e, também, permite que os investidores ajustem suas opções de portfólio ao considerar os produtos dos bancos islâmicos de acordo com as especificidades regionais.

Palavras-Chave: Finanças Islâmicas. Ambiente Geográfico. Dados em Painel. Regressão Quantílica.

Editado em português e inglês. Versão original em Português.

Recebido em 8/1/2021. Revisado em 29/3/2021. Aceito em 15/4/2021 pelos Prof. Dr. Sérgio Murilo Petri (Editor-Chefe) e Prof. Dr. Sandro Vieira Soares (Editor Adjunto). Publicado em 7/5/2021.

Copyright (C) 2021 RCCC. Todos os direitos reservados. É permitida a citação de parte de artigos sem autorização prévia, desde que identificada a fonte. 


\begin{abstract}
The main goal of this study is to examine the influence of the geographic environment on the performance of Islamic banks present in four regions: Africa, Asia, Europe and North America. To achieve this goal, we have used daily data from 113 Islamic banks between 2010 and 2019. We applied different methodological approaches, such as principal component analysis and quantile regression with fixed effects for panel data. As a result, we found that the analysis of the main components shows that the performance of Islamic banks varies between regions. The regression of the linear panel highlights that the geographic environment positively and significantly affects the Islamic banking system, suggesting the importance of this aspect's effects. Finally, the environmental effect seems to vary with the quantiles, bringing positive effect to the lower quantile and negative effect to the highest quantile. This quantile specification points to the environment-performance nonlinear relationship of the Islamic bank, reflecting a discipline variable in the time imposed by the Council of Shariah or Islamic Law. This discovery helps to better explain the main difference between Islamic banks in the East and those in the West, and also allows investors to adjust their portfolio options when considering the products of Islamic banks according to regional specificities.
\end{abstract}

Keywords: Islamic Finance. Geographic Environment. Panel Data. Quantile Regression.

\title{
1 INTRODUÇÃO
}

A característica central dos bancos islâmicos (BIs) é a proibição do pagamento e recebimento de juros (ou riba). A definição convencional de riba é a proibição de cobrança de juros ao emprestar dinheiro e de qualquer acréscimo injustificado (como multas). Emprestar dinheiro cobrando juros permite ao credor aumentar seu capital sem nenhum esforço, porque o dinheiro por si só não cria valor agregado. Além disso, as finanças islâmicas proíbem o investimento em transações que envolvam jogos de azar, álcool e drogas, e transações incluindo incerteza quanto ao assunto e aos termos dos contratos (ou gharar). Esta prática inclui a proibição de vender algo que não seja de sua propriedade ou cujas características sejam incertas e sob condições contratuais ambíguas. Nas finanças islâmicas, o capital deve ter um propósito social e ético além do retorno puro e irrestrito. Além disso, a associação entre risco-retorno e a noção de participação nos lucros e perdas e parcerias inerentes aos contratos islâmicos são centrais para as finanças islâmicas.

O sistema bancário islâmico foi desenvolvido originalmente na década de 1970 para proteger o produto da produção de petróleo nos países do Golfo. No entanto aumentou rapidamente nos últimos anos, não apenas nesta região, mas também globalmente. Enquanto alguns países adotaram totalmente o sistema bancário islâmico como principal sistema bancário, em outros países o sistema bancário islâmico foi parcialmente introduzido em paralelo com os bancos convencionais. Para ilustrar esse rápido crescimento do setor bancário islâmico, é importante observar que seus ativos totalizaram cerca de US\$ 2 trilhões em 2018. Além disso, de acordo com o Relatório de Competitividade do Setor Islâmico Mundial (Ernst \& Young (EY), 2020), o setor bancário islâmico tem crescido mais rápido que o setor bancário convencional ou tradicional, a uma taxa anual de $17,6 \%$ entre 2009 e 2018 , e projeta-se um crescimento médio anual de 19,7\% até 2021. Dessa forma, os BIs são regidos por um sistema que regula e opera as atividades bancárias sob a economia islâmica, consistente com os princípios da Shariah (Lei Islâmica). Consequentemente, o sistema bancário islâmico também é chamado de sistema em conformidade com a Shariah. A aplicação dessas regras religiosas visa reduzir a assunção de riscos e criar um sistema financeiro mais ético e moral (Lewis \& Algaoud, 2001; Kabir, Worthington \& Gupta, 2015). 
Enquanto estudos precedentes relacionados tendem a realizar análises quantitativas, a principal contribuição deste estudo refere-se à proposição de uma análise híbrida incorporando algumas dimensões qualitativas para avaliar o desempenho financeiro dos BIs. Nesse sentido, este estudo responde à seguinte pergunta: $O$ ambiente geográfico afeta o desempenho dos bancos islâmicos? A hipótese investigativa gira em torno de entender se o negócio associado aos produtos financeiros islâmicos em um país muçulmano é diferente daquele em um país não muçulmano. Os negócios bancários também variam entre os países muçulmanos porque atingem altos níveis quando apenas os BIs existem em um país, enquanto são menos significativos nos países em que coexistem bancos convencionais e BIs. Além disso, o sistema bancário islâmico mostra diferentes desempenhos regionais dependendo da presença de uma autoridade financeiro/bancária oficial (Kabir, Worthington \& Gupta, 2015; Jawadi, Jawadi, Ben Ameur, \& Idi Cheffou, 2017). Os processos de seleção de empresas elegíveis com relação às regras da Sharia também variam em nível regional e por país. Além disso, outros fatores como falta de transparência, imagem do BI e confusão entre questões jurisprudenciais islâmicas podem afetar os BIs de maneira diferente.

Dessa forma, o sistema bancário islâmico exige mais investigações e pesquisas, dado seu rápido desenvolvimento nas últimas duas décadas no Oriente Médio, bem como na Europa e nos Estados Unidos. Em particular, é importante especificar os fatores de desempenho dos Bis para melhor informar os investidores e os formuladores de políticas sobre suas especificidades, oportunidades e perspectivas globais (Jawadi et. al, 2017). O foco no desempenho é particularmente importante, pois nos permite estudar o retorno e o risco financeiro, que são cruciais para os investidores. A análise de desempenho também é necessária para melhorar a avaliação e classificação dos preços dos ativos. Por outro lado, a investigação regional de desempenho ajuda a especificar rigorosamente os fatores regionais que os investidores devem levar em consideração ao investir nos produtos de um banco islâmico. Portanto este estudo se concentra no desempenho dos BIs, ao mesmo tempo em que esclarece as conclusões inconclusivas e os resultados mistos da literatura precedentes sobre BIs e ambiente geográfico. Não é objetivo desta pesquisa comparar o sistema bancário islâmico com o sistema convencional. Em vez disso, verificamos se essa heterogeneidade dos achados está associada à presença de diferentes fatores culturais, geográficos e religiosos que podem afetar os BIs de maneira diferente.

A quantificação desse efeito é realizada usando uma especificação de dados em painel não linear, permitindo capturar o efeito do ambiente por quantil. Além disso, o uso de dimensões de painel e tempo nos permite modelar uma amostra de dados mais robusta (Jawadi et. al, 2017). No contexto de uma aparente falta de consenso na literatura sobre o crescimento do sistema bancário islâmico, este estudo faz uma contribuição valiosa ao tentar modelar e capturar as não linearidades inerentes no desempenho dos BIs em locais geográficos distintos.

\section{REVISÃO DA LITERATURA}

Em função da última crise financeira global (2008-2009), os bancos convencionais sofreram grandes perdas e os BIs foram considerados capazes de proteger os investidores de grandes perdas em momentos de incertezas financeiras. Como tal, o sistema bancário islâmico tornou-se objeto de vários estudos empíricos com foco em retornos, riscos e desempenho bancários. No entanto esses estudos não chegaram a conclusões unânimes sobre a influência do ambiente no desempenho dos BIs. Por um lado, estes parecem melhorar o crescimento econômico e a eficiência macroeconômica de determinado país (Imam \& Kpodar, 2010; Gheeraert \& Weill, 2015; Imam \& Kpodar, 2016). Verificou-se que os BIs são mais eficientes, com melhor desempenho e menor risco quando comparados aos bancos convencionais, devido a sua adesão a uma referência religiosa e a regras éticas, bem como à proibição de excesso de risco 
e especulação (Lewis \& Algaoud, 2001). Além disso, o recente efeito da última grande crise financeira global parece ter sido menos oneroso para os BIs do que para os bancos convencionais, dadas as características naturais de aversão ao risco dos produtos dos BIs (Fakhfakh, Hachicha, Idi Cheou \& Selmi, 2016). Por outro lado, alguns estudos mostram que os BIs diferem pouco dos bancos convencionais, descobrindo que os dois tipos de bancos estão indiretamente em concorrência (Jawadi, Cheffou, \& Jawadi, 2016b). Além disso, os BIs foram criticados por não serem suficientemente éticos e transparentes para fornecer um modelo típico com o qual liderar bancos convencionais (Imam \& Kpodar, 2010; Jawadi, Cheffou, \& Jawadi, 2016a).

O surgimento do sistema bancário islâmico no cenário financeiro global atraiu rapidamente a atenção acadêmica, e a lista de periódicos que avaliam empiricamente o desempenho dos BIs está se expandindo. A literatura inicial concentra-se principalmente em medir a eficiência dos BIs. Em seguida, evoluiu para a identificação dos determinantes da eficiência do BI e, posteriormente, se estende a outras métricas ou medidas de desempenho do banco, principalmente avaliadas em comparação com os bancos convencionais (Hassan \& Aliyu, 2018; Narayan \& Phan, 2019). Por exemplo, Beck, Jonghe e Schepens (2013), Kabir e Worthington (2017), Zins e Weill (2017) e Ibrahim e Rizvi (2018) comparam o risco e a estabilidade de bancos islâmicos e convencionais. Enquanto isso, Olson e Zoubi (2017) e Yanikkaya, Gumus e Pabuccu (2018) examinam comparativamente a lucratividade de ambos os tipos de bancos. A eficiência dos BIs em comparação com os bancos convencionais é avaliada nos estudos de Beck e Hesse (2009), Imam \& Kpodar, 2010 e Abdul-Majid, Manizheh \& Mansor (2017) e Alqahtani, Mayes e Brown (2017).

Na prática, os produtos dos BIs parecem, a priori, menos arriscados do que os bancos convencionais devido à aplicação dos princípios da Shariah (por exemplo, proibição de especulação, proibição de taxas de juros). No entanto essa conclusão é questionável, porque a maioria dos bancos-padrão suporta apenas o risco bancário, enquanto os BIs suportam o risco bancário padrão e um risco específico adicional associado às características dos contratos financeiros islâmicos. Isso adiciona mais ambiguidade ao considerar os BIs. Por todas essas razões, os níveis de desempenho e eficiência dos BIs continuam incertos, apesar do importante número de estudos empíricos relacionados. Além disso, os resultados desses estudos empíricos variam de acordo com os dados da amostra e dos períodos considerados (Fakhfakh et al., 2016) e com o número de países analisados (Jawadi et. al, 2017).

Utilizando dados bancários de 22 países muçulmanos, Abedifar, Hasan e Tarazi (2016) verificam os benefícios potenciais da presença de BIs nos bancos convencionais desses países. Ou seja, os bancos convencionais que operam em países predominantemente muçulmanos registram melhorias em sua eficiência de custos quando também há grandes BIs operando no mesmo país. Arouri, Ben Ameur, Jawadi, Jawadi \& Louhichi (2013) e Jawadi et. al (2017) fornecem mais suporte para as influências da presença de BIs no comportamento bancário convencional. Mais especificamente, de acordo com Arouri et al. (2017) e Jawadi et. al (2017) as taxas de depósito dos bancos convencionais tendem a ser mais altas e, portanto, beneficiam os depositantes em países onde há forte presença de BIs.

\section{METODOLOGIA}

Utilizamos dados diários para o período de julho de 2010 a abril de 2019, obtidos da Bloomberg. Os dados incluem o preço de fechamento para os 113 BIs estabelecidos em quatro regiões (Ấfrica, Ásia, Europa e Estados Unidos): International Bank of Kuwait, no Egito (IBKE); Faisal Islamic Bank, no Egito (FIBE); Al-Baraka, no Egito (ABE); Banco Islâmico da Jordânia (BIJ); Commercial Bank, no Kuwait (CBK); Bank Muscat International, no Bahrein (BMIB); Qatar International Bank (QIIBD); Banco Al-Rajhi, na Arábia Saudita (ARBSA); 
Banco Islâmico de Abu Dhabi (BIAD); Banco Islâmico de Dubai (DIB); Banco Islâmico Europeu, no Reino Unido (EIIBUK); e Albaraka Partners, nos Estados Unidos (APUSA). A amostra é suficientemente heterogênea e inclui os principais bancos, o que garantiu a cobertura das principais atividades dessas instituições em todo o mundo. No entanto a amostra foi limitada devido à indisponibilidade dos dados. Consequentemente, não foi possível identificar o mesmo número de BIs por região. Também incluímos o preço do petróleo West Texas Intermediate (WTI) como variável de controle. Isso nos permite verificar a ligação entre os preços do petróleo e os bancos islâmicos seguindo estudos de Jawadi et. al (2017).

Além disso, usamos uma proxy para medir o desempenho dos BIs. A seleção da proxy para desempenho foi orientada de acordo com a disponibilidade dos dados. Por conseguinte, em primeiro lugar, usamos duas proxies: Q de Tobin e Retorno sobre Ativos (ROA), e executamos testes para as duas proxies. No entanto, como os resultados foram muito próximos, mantivemos apenas os do Q de Tobin. O Q de Tobin representa a razão entre o valor de mercado das ações existentes de um BI e o custo de reposição de seus ativos físicos. A vantagem do Q de Tobin é que ele leva em consideração a taxa de juros e o retorno esperado (p.ex., Lindenberg \& Ross, 1981; Hayashi, 1982; Skinner, 1993; Chung e Pruitt, 1994). Finalmente, para fazer a proxy da variável de ambiente geográfico, observada como $A m b$ na Equação (1), e medir seu efeito no desempenho dos BIs, criamos uma variável dummy e definimos a variável de ambiente da seguinte maneira:

$$
A m b_{t}=\left\{\begin{array}{c}
1 \text { se o IB estiver no Ocidente } \\
0 \text { caso contrário }
\end{array}\right]
$$

Em outras palavras, a variável de ambiente assume dois valores que variam de acordo com a localização do BI em consideração. Será 1 se o banco estiver no Oriente (África e Ásia) e 0 se o banco estiver no Ocidente (Europa e Estados Unidos). Na primeira região, os BIs estão principalmente nos países muçulmanos, enquanto no Ocidente o Islã não é a religião principal e a cultura também é diferente da do Oriente. Consequentemente, a equação (1) permite capturar o efeito do ambiente variável no tempo e por região. Portanto essa escolha captura indiretamente as diferentes dimensões (por exemplo, cultura, religião, regulamentos e lei, tradições da sociedade, crenças) que variam de acordo com a região. Essa especificação nos permite verificar se a heterogeneidade ambiental afeta o desempenho financeiro dos bancos.

Em seguida, para obter uma visão geral do desempenho entre os 113 bancos, relatamos suas principais estatísticas descritivas na Tabela 1. Essas estatísticas foram obtidas usando o Q de Tobin. A Tabela 1 mostra que, para todos os BIs considerados, o Q de Tobin médio está acima de 1, exceto para os bancos FIBE e EIIBUK. Tal fato indica que investimentos adicionais na maioria dos bancos faria sentido, pois os lucros gerados excederiam o custo dos ativos do banco. Encontramos também um excesso de volatilidade importante para o Q de Tobin na maioria dos BIs, particularmente APUSA (16\%) e ARBSA (18\%), implicando uma variação significativa no desempenho desses bancos. Esse excesso de volatilidade pode ser justificado pelo fato de que, durante esse período, os retornos dos BIs foram mais variáveis devido, talvez, ao efeito da crise financeira global, crise de liquidez dos bancos convencionais e desaceleração econômica. Exceto pelos bancos DIB e APUSA, um excesso de curtose (leptocúrtica) importante caracteriza as distribuições dos outros bancos. Finalmente, a normalidade é fortemente rejeitada para todas as séries. As séries em consideração também mostram assimetria positiva, indicando que a distribuição é inclinada para a direita com uma cauda superior mais longa do que uma cauda inferior. 
Tabela 1

Estatísticas descritivas do Q de Tobin

\begin{tabular}{|c|c|c|c|c|c|c|c|c|c|c|c|c|}
\hline & IBKE & FIBE & $\mathrm{ABE}$ & JBI & CBK & BMIB & QIIBD & ARBSA & BIAD & $\overline{\text { DIB }}$ & EIIBUK & APUSA \\
\hline Sign. & 1,06 & 0,99 & 1,01 & 1,06 & 1,10 & 1,04 & 1,18 & 1,32 & 1,04 & 1,05 & 0,61 & 1,35 \\
\hline Média & 1,02 & 0,98 & 1,00 & 1,05 & 1,06 & 1,02 & 1,16 & 1,28 & 1,02 & 1,02 & 0,60 & 1,36 \\
\hline Máx. & 1,34 & 1,14 & 1,09 & 1,15 & 1,46 & 1,33 & 1,71 & 2,21 & 1,19 & 1,27 & 0,96 & 1,76 \\
\hline Mín. & 0,97 & 0,97 & 0,98 & 1,02 & 1,02 & 0,97 & 1,11 & 1,13 & 0,99 & 0,96 & 0,51 & 0,98 \\
\hline Desv.P. & 0,09 & 0,04 & 0,03 & 0,03 & 0,09 & 0,08 & 0,12 & 0,17 & 0,04 & 0,08 & 0,10 & 0,13 \\
\hline Skew & 1,96 & 2,67 & 1,99 & 1,43 & 2,56 & 2,76 & 2,63 & 1,99 & 1,89 & 1,02 & 1,56 & 0,16 \\
\hline Kurt & 6,68 & 9,76 & 5,93 & 5,90 & 9,03 & 10,14 & 9,27 & 9,25 & 6,69 & 3,45 & 5,84 & 3,77 \\
\hline $\mathrm{J}-\mathrm{B}$ & 0,00 & 0,00 & 0,00 & 0,00 & 0,00 & 0,00 & 0,00 & 0,00 & 0,00 & 0,00 & 0,00 & 0,00 \\
\hline Obs. & 3.153 & 3.153 & 3.153 & 3.153 & 3.153 & 3.153 & 3.153 & 3.153 & 3.153 & 3.153 & 3.153 & 3.153 \\
\hline
\end{tabular}

Nota. Obs indica o número de observações.

Max e Min se referem aos valores máximo e mínimo de Q de Tobin, respectivamente.

Med, Desv. P. Skew, Kurt denotam o valor mediano, desvio-padrão, assimetria e curtose, respectivamente.

Em seguida, calculamos a matriz de correlação para fornecer uma visão geral de outros vínculos entre o desempenho e o investimento dos BIs. A Tabela 2 mostra a forte correlação entre os BIs, considerando as razões do Q de Tobin. Importante notar que, embora a medida de desempenho para BIs nos Estados Unidos esteja menos relacionada à medida de desempenho, para outros BIs há mais evidências de altos vínculos regionais. Para os pares (IBKE, BIAD), (IBKE, ARBSA), (BIJ, BMIB), (BMIB, ARBSA) e (CBK, QIIBD), as correlações bilaterais excedem $60 \%$ e atingem $91 \%$ para o último par. No geral, isso indica que, a priori, os bancos que evoluem em regiões semelhantes compartilham fatores culturais, sociais e ambientais comuns que indiretamente afetam seu desenvolvimento e desempenho da mesma maneira.

Tabela 2

Matriz de Correlação

\begin{tabular}{lcccccccccccc}
\hline & IBKE & FIBE & ABE & BIJ & CBK & BMIB & QIIBD & ARBSA & BIAD & DIB & EIIBUK & APUSA \\
\hline IBKE & 1,00 & 0,11 & 0,66 & 0,71 & 0,81 & 0,82 & 0,75 & 0,86 & 0,20 & 0,41 & 0,55 & 0,02 \\
FIBE & & 1,00 & 0,23 & 0,31 & 0,28 & 0,20 & 0,27 & 0,05 & 0,22 & 0,37 & 0,13 & 0,22 \\
ABE & & & 1,00 & 0,66 & 0,80 & 0,73 & 0,87 & 0,52 & 0,37 & 0,55 & 0,75 & 0,33 \\
BIJ & & & & 1,00 & 0,80 & 0,60 & 0,84 & 0,48 & 0,36 & 0,70 & 0,40 & 0,16 \\
CBK & & & & & 1,00 & 0,90 & 0,91 & 0,72 & 0,51 & 0,77 & 0,75 & 0,37 \\
BMIB & & & & & 1,00 & 0,81 & 0,86 & 0,39 & 0,58 & 0,73 & 0,39 \\
QIIBD & & & & & & 1,00 & 0,66 & 0,47 & 0,68 & 0,71 & 0,27 \\
ARBSA & & & & & & & 1,00 & 0,11 & 0,25 & 0,51 & 0,09 \\
BIAD & & & & & & & & 1,00 & 0,67 & 0,37 & 0,42 \\
DIB & & & & & & & & & 1,00 & 0,55 & 0,59 \\
EIIBUK & & & & & & & & & & 1,00 & 0,30 \\
APUSA & & & & & & & & & & & & 1,00 \\
\hline
\end{tabular}

Em resumo, essa análise preliminar sugere diferentes delineamentos para os bancos islâmicos, ao considerar fatores geográficos. Essa ferramenta, no entanto, assume que o conjunto de dados é uma combinação linear das variáveis. Assim, estendemos essa abordagem para testar diretamente o efeito do ambiente geográfico nos BIs, considerando várias especificações lineares e não lineares para melhor capturar a dinâmica e os direcionadores do desempenho do BI.

\section{ANÁLISE DOS RESULTADOS}

Apresentaremos primeiro a especificação linear de dados em painel, usada para avaliar o efeito do ambiente geográfico no desempenho dos BIs. Segundo, apresentamos a abordagem de regressão quantílica para verificar outros efeitos assimétricos. Estima-se que ambos os modelos 
investiguem a dinâmica de desempenho dos BIs entre as regiões. Curiosamente, é necessário o uso de uma estrutura de dados em painel para considerar mais heterogeneidade entre os BIs.

\subsection{Especificação de dados do painel linear}

Investigamos o efeito do ambiente geográfico no desempenho de BIs em uma estrutura linear univariada. Para esse fim, estimamos o seguinte modelo de dados em painel fixo:

$$
Q T_{i, t}=\propto_{i, 0}+\beta \propto A m b_{i, t}+\varepsilon_{i, t}
$$

Onde:

$\propto_{i, 0}$ e $\beta$ são parâmetros do regressor,

$\varepsilon i, t \quad \rightarrow$ i.i.d. $\left(0, \sigma^{2}\right), \forall t=1, \ldots, T$ e $i=1, \ldots . ., N Q T$ denota o $\mathrm{Q}$ de Tobin,

$A m b$ define a variável de ambiente,

$\mathrm{N}$ indica o número de Bancos Islâmicos (BIs).

Estimamos a Equação 2 e relatamos os principais resultados da Tabela 3. Esses resultados mostram que o ambiente geográfico afeta de forma significativa e positiva o desempenho e, portanto, o desenvolvimento de BIs. Esse resultado sugere que os BIs no Oriente não beneficiam de externalidades naturais e positivas em comparação com os BIs no Ocidente.

A regressão 2 tem como objetivo avaliar os efeitos do ambiente. Uma variável de controle associada aos retornos de óleo é adicionada para estender a regressão 2, para verificar a validade dos resultados disso. A seguir, chamamos esse modelo de regressão estendida 3 . Curiosamente, o modelo 3 valida nossos achados anteriores. De fato, os resultados do modelo 2 permanecem robustos, mesmo quando estimamos o modelo 3. Quanto ao efeito do petróleo, o modelo 3 mostra que isso é positivo e significativo, sugerindo uma reação positiva da indústria de petróleo para as atividades dos BIs. Embora esse achado confirme que os BIs surgiram para proteger os recursos do mercado de petróleo, o efeito do petróleo é, contudo, baixo (abaixo de $4 \%$ ). Isso é explicado pelo fato de que, durante o período considerado neste estudo, os preços do petróleo aumentaram e os benefícios do petróleo diminuíram. Mesmo ao considerar outras procurações para os preços do petróleo, os resultados permanecem inalterados. Além disso, para verificar a robustez de nossa especificação, verificamos que a regressão 2 tem como objetivo avaliar os efeitos do ambiente. Uma variável de controle associada aos retornos de óleo é adicionada para estender a colinearidade da regressão 2 entre as variáveis explicativas, novamente usando o ACP. Nossos resultados mostram que os primeiros autovalores são estatisticamente diferentes de zero, sugerindo um índice de condição fraco e, portanto, a ausência de colinearidade.

Tabela 3

Estimativa da regressão de dados do painel linear

\begin{tabular}{lcc}
\hline Coeficientes & Modelo 2 & Modelo 3 \\
\hline Constante & $-0,32^{* * *}$ & $-0,47 * * *$ \\
& $(0,00)$ & $(0,00)$ \\
$\boldsymbol{\beta}$ & $0,44 * * *$ & $0,43 * * *$ \\
& $(0,00)$ & $(0,00)$ \\
$\boldsymbol{Y}$ & - & $0,03 * * *$ \\
$\mathbf{R}^{2}$ & - & $(0,00)$ \\
LL & 0,59 & 0,61 \\
Prob. (F - Test) & $10.057,64$ & $10.105,34$ \\
\hline
\end{tabular}

Nota. $\mathrm{R}^{2}$ e LL denotam o quadrado $\mathrm{R}$ ajustado e a probabilidade logarítmica, respectivamente valores em (.) referem-se a valores-p robustos. (***) indica significância ao nível de $1 \%$.

Teste F indica o Teste Fisher. 
Embora essa conclusão implique um efeito positivo e significativo do ambiente geográfico sobre o desempenho financeiro dos BIs, a estrutura em consideração apenas captura o efeito linear em torno da média. A estrutura não pode reproduzir o efeito ambiental quando os preços dos produtos dos BIs sofrem alta volatilidade e mudanças significativas. Portanto estendemos o modelo (3) e focamos na regressão quantílica, que foi introduzida por Koenker e Bassett (1978) e desenvolvida por Koenker e Hallock (2001) e Koenker (2005). Isso tem a vantagem de considerar uma maior assimetria e calcular o efeito ambiental em torno de diferentes Como as normas ambientais das BIs (regras da Shariah, critérios de seleção ética, reputação e consideração dos BIs, aspectos culturais, análise dos produtos dos BIs) evoluem ao longo do tempo, assim como o desempenho financeiro e o tamanho da IB, o efeito do ambiente geográfico pode seguir um padrão não linear que pode escapar da modelagem linear. Portanto adotamos uma regressão quantil para analisar a relação entre o ambiente e o desempenho da IB. Em particular, o efeito ambiental pode variar ao longo do tempo. De fato, as normas ambientais apresentadas podem mostrar incômodos que afetam de maneira diferente e temporal a atividade dos BIs. Por exemplo, o efeito pode variar com a fase do ciclo de negócios (crise versus período calmo) e ser assimétrico. Além disso, qualquer mutação ou notícia que chegue ao mercado pode afetar a sociedade e seu ambiente. Para explicar adequadamente essas mudanças nos efeitos ambientais, uma estrutura econométrica acessível, como o modelo de regressão quantílica, tornase necessário.

\subsection{A Especificação de Regressão Quantil}

Aqui, testamos se o impacto do meio ambiente no setor bancário islâmico é mais forte (ou mais fraco) quando o desempenho do IB é extraordinariamente alto (ou baixo). Por fim, especificamos uma regressão quantílica de painel linear da seguinte maneira:

$$
q_{\propto}\left(Q T_{i, t} \mid \Omega_{t}\right)=\text { constante }+\Phi_{1, \alpha} A m b_{i, t}+\Phi_{2, \alpha} R o_{i, t}
$$

Onde:

$\Omega_{t}$ denota todas as informações disponíveis no momento t,

$\Phi_{1, \alpha}$ e $\Phi_{2, \alpha}$ são os coeficientes de declive que variam por quantil,

$q_{\propto}\left(Q T_{i, t} \mid \Omega_{t}\right)$ define o quantil condicional.

Assim, a Equação (3) avalia o efeito ambiental ao estimar a distribuição do desempenho do IB. Tecnicamente, os parâmetros do modelo de previsão quantil são estimados substituindo a função de perda quadrática pela seguinte função de perda de escala:

$$
L \propto\left(e_{i, t}+1\right)=\left(\propto-1\left\{e_{i, t}+1<0\right\}\right) e_{i, t}+1
$$

Onde:

$e_{i, t}=Q T_{i, t}-q_{\alpha, i, t}$ denota o erro de previsão

$q_{\alpha, i, t}=q_{\alpha}\left(Q T_{i, t} \mid \Omega_{t}\right)$ é a previsão quantílica condicional no tempo t $1\left\{e_{i, t}+1<0\right\}$ é uma função indicadora

Como em Jawadi e Sousa (2014), os intervalos de confiança são calculados usando uma inversão de um teste de classificação. Consequentemente, a condição de primeira ordem associada à minimização do valor esperado de (4) em relação às previsões, $q_{\alpha, i, t}$ corresponde ao quantil da distribuição Q de Tobin. Koenker (2005) mostra que a previsão ideal é o quantil 
condicional $q_{\alpha, i, t}=F^{-1}(\alpha)$, em que $\mathrm{F}_{\mathrm{t}}$ é a função de distribuição condicional de $\mathrm{Q}$ de Tobin. Estimamos a Equação (3) para diferentes quantis e relatamos na Tabela 4 os principais resultados associados ao efeito do ambiente geográfico por quantil.

A Tabela 4 fornece alguns resultados interessantes. Primeiro, quando o desempenho dos BIs é próximo da mediana, o efeito do ambiente geográfico, embora positivo, é próximo de zero. Segundo, o efeito ambiental é particularmente diferente quando a distribuição do desempenho do IB é próxima das caudas da distribuição. Observamos um grande coeficiente quando o desempenho do IB está próximo dos quantis de $2,5 \%$ e $25 \%$. Os coeficientes para esses dois quantis $(0,65$ e 0,50$)$ são maiores que os coeficientes da estimativa do OLS $(0,43)$. No entanto, quando o desempenho do IB está próximo dos quantis $75 \%$ e 97,5\%, os efeitos ambientais são negativos.

Tabela 4

\section{Estimativa da regressão quantílica}

\begin{tabular}{lc}
\hline Quantil & Efeito Ambiental $\left(\Phi_{\mathbf{1}, \boldsymbol{\alpha}}\right)$ \\
\hline $2,5 \%$ & $0,65^{* * *}$ \\
$25 \%$ & $0,50^{* * *}$ \\
$50 \%$ & $0,06^{* * *}$ \\
$75 \%$ & $-0,21^{* * *}$ \\
$97,5 \%$ & $-0,16^{* * *}$ \\
OLS & $0,43^{* * *}$ \\
Wald Test (p-value) & $0,00^{* * *}$ \\
Koenker and Xia (2006) Test (p-value) & $0,00^{* * *}$ \\
\hline
\end{tabular}

Nota. (***) indica significância no nível de 1\%. OLS denota a estimativa do modelo (3)

Assim, esses achados também confirmam a não linearidade inerente ao efeito do ambiente geográfico nos bancos islâmicos. Desse modo, concluímos que o efeito do ambiente geográfico no desempenho do IB é negativo na cauda direita da distribuição do desempenho da IB, enquanto é positivo na cauda esquerda. Isso é explicado pelo fato de que, para baixos níveis de desempenho, o ambiente estimularia e promoveria as atividades de IB; no entanto, quando os BIs atingem um alto nível de desempenho, a diretoria da Shariah intensifica o controle e ativa regras para impedir que os BIs busquem objetivos de negócios. Além disso, isso implica que, no ponto mais baixo da cauda da distribuição, o ambiente geográfico está positivamente correlacionado com o desempenho do IB, enquanto no ponto mais alto da cauda o efeito do ambiente é negativo.

Embora esses resultados apontem apenas para os efeitos significativos do ambiente sobre o desempenho dos BIs ao considerar os quantis, aplicamos dois testes: o teste de Wald (teste de igualdade da inclinação quantil) e o teste de Koenker e Xiao (2006), que também calcula um teste conjunto para verificar se todos os efeitos covariáveis satisfazem a hipótese nula da igualdade dos coeficientes de inclinação entre países. Ambos os testes rejeitam significativamente a hipótese nula, sugerindo que o efeito do ambiente no desempenho dos BIs varia com os quantis. Consequentemente, esses resultados sugerem a presença de variação no tempo no efeito ambiental no desempenho da IB e implicam que a relação de desempenho meio ambiente - BIs mostre assimetria, e não linearidade.

Em outras palavras, parece que o controle das autoridades do conselho da Shariah varia de acordo com o nível de desempenho de um IB, gerando uma disciplina variável no tempo para controlar os BIs. De fato, quando o IB faz um pequeno retorno e mostra desempenho fraco, a ação do controle do conselho da Shariah não é ativada; pelo contrário, para um alto nível de desempenho, a Shariah pode ser mais agressiva e recordar fortemente as dimensões morais, éticas, espirituais e sociais que um IB deve mostrar para pressioná-lo a reduzir seu investimento 
e manter a Shariah em conformidade projetos no mínimo. Essa ação pode justificar o efeito negativo do ambiente no desempenho da IB na cauda esquerda. Além disso, o fato de o nível dessa disciplina e da aplicação das regras da Shariah variar de um país para outro pode justificar a assimetria e a não linearidade inerentes ao relacionamento de desempenho e ambiente do banco. Essa assimetria também pode ser explicada pela presença de diferentes escolas bancárias islâmicas ou estudiosos, que nem sempre fornecem opiniões unânimes para produtos e empresas de BIs, gerando diferentes maneiras de investir em BIs.

No geral, esse achado sugere maior não linearidade e assimetria na relação entre o desempenho do IB e o ambiente geográfico. Os resultados confirmam que o desempenho do IB varia significativamente entre as regiões. Contudo, como mostra Canay (2011) e Jawadi et. al, 2017 ao considerar os dados do painel de regressão quantil, a consistência dos estimadores pode ser importante. De fato, Canay (2011) fornece um conjunto de condições suficientes para identificar um modelo de regressão quantílica com efeitos fixos. $\mathrm{O}$ autor mostra que, como n (o número de pessoas) e $\mathrm{T}$ (período aumentado), o estimador obtido é consistente e assintoticamente normal. Em nosso estudo, os dados são diários e o período é suficientemente alto, mas o número de bancos é relativamente pequeno $(\mathrm{n}=113)$, portanto a consistência do estimador deve ser analisada com cautela. Porém é importante notar que essa regressão quantílica supera o modelo linear, pois fornece uma análise quantílica mais aparente, menos sensível a discrepantes e distribuições alongadas.

\section{CONSIDERAÇÕES FINAIS}

Este estudo investigou o efeito do ambiente geográfico no desempenho dos BIs. Nossa amostra abrangeu 113 bancos presentes em quatro regiões (África, Ásia, Europa e Estados Unidos) durante os anos de 2010 a 2019. Como conclusões, tivemos que, primeiro, uma análise preliminar de séries temporais apontou evidências de heterogeneidade no desempenho financeiro desses BIs. Segundo, o ACP confirmou essa heterogeneidade e mostrou que os BIs no Oriente diferem dos BIs no Ocidente. Em seguida, avaliamos o efeito direto do ambiente geográfico no desempenho da IB, usando uma regressão linear de dados em painel e regressão quantílica. Enquanto a regressão linear apontou para um efeito positivo e significativo, a regressão quantílica mostrou que o efeito ambiental varia com os quantis, sugerindo mais evidências de assimetria e não linearidade. O efeito ambiental é positivo (negativo) e significativo para a distribuição mais baixa (mais alta) da cauda do desempenho do BI. Isso é uma indicação de assimetria, não linearidade e complexidade no relacionamento entre os BIs e o ambiente geográfico.

Esses achados têm duas importantes implicações econômicas e políticas. Primeiro, nossos resultados ajudam os investidores a identificar os BIs com melhor desempenho em cada região. Segundo, nossos resultados esclarecem os efeitos do ambiente geográfico nas atividades dos BIs e ajudam os estudiosos a especificar essas externalidades. Além disso, embora esses resultados permitam aos investidores melhorar suas carteiras, incluindo os produtos de BIs, com base na região em consideração, nossa conclusão é útil para levar em conta os efeitos dessas externalidades para melhorar o sistema bancário islâmico.

Para resumir, este estudo focou na relação desempenho-ambiente para os BIs. Portanto não incluímos bancos convencionais, mas eles podem ser incluídos em modelos futuros. Ele mostrou que o ambiente geográfico afeta os BIs e quantificai esse efeito, mas não identificou seus componentes (cultura, religião, lei, regulamentos), o que pode ser uma extensão adicional. Além disso, nossos resultados não implicam que os BIs no Ocidente (Europa e Estados Unidos) não sejam promissores, mas que o desempenho dos BIs pode variar por região de acordo com sua localização e ambiente. Uma extensão futura adicional seria verificar quais fatores impulsionam esse efeito ambiental. Para fazer isso, seriam necessários dados quantitativos e 
qualitativos sobre cultura, religião, regulamentação e negócios, para uma grande amostra de BIs em diferentes regiões do mundo. Além disso, observar a variação subjacente nos BIs do oriente também é um tópico de pesquisa interessante que pode nos permitir identificar se as diferenças culturais/ambientais entre os BIs no Oriente e no Ocidente podem impulsionar o desempenho dos BIs.

\section{REFERÊNCIAS}

Abedifar, P., Hasan, I., \& Tarazi, A. (2016). Finance-growth nexus and dual-banking systems: Relative importance of Islamic banks. Journal of Economic, Behavior \& Organization, 132 (3), 198-215.

Abdul-Majid, M., Manizheh, F., \& Mansor, J. (2017). Performance of Islamic and conventional banks: A meta-frontier approach. Research in International Business and Finance, 42(3), $1327-1335$.

Alqahtani, F., Mayes, D. G., \& Brown, K. (2017). Islamic Bank Efficiency Compared to Conventional Banks During the Global Crisis in the GCC Region. Journal of International Financial Markets, Institutions and Money, 51, 58-74.

Arouri, M., Ben Ameur, H., Jawadi, N., Jawadi, F., \& Louhichi, W. (2013). Are Islamic performance innovations enough for investors to escape from a financial downturn? Further evidence from portfolio simulations. Applied Economics, 45(24), 3412-3420.

Beck, T., De Jonghe, O., \& Schepens, G. (2013). Bank Competition and Stability: Cross-Country Heterogeneity. Journal of Financial Intermediation, 22, 218-244.

Beck, T., \& Hesse, H. (2009). Why Are Spreads so High in Uganda? Journal of Development Economics, 88, 192-204.

Canay, I. A. (2011). A simple approach to quantile regression for panel data. The Econometrics Journal, 14(3), 368-386.

Chung, K. H., \& Pruitt, S. W. (1994). A simple approximation of Tobin's q. Financial management, 70-74.

Ernst \& Young (EY) (2020). World Islamic Banking Competitiveness Report 2019-20. Recuperado em 12 abril, 2020, de http://www.ey.com/Publication/vwLUAssets/ey-worldislamic-banking-competitiveness-report-2020/\%24FILE/ey-world-islamic-bankingcompetitiveness-report-2020.pdf

Fakhfakh, M., Hachicha, N., Idi Cheou, A., \& Selmi, N. (2016). Measuring volatility persistence for conventional and Islamic banks: A FI-EGARCH approach. Emerging Markets Review, 27, 84-99.

Gheeraert, L., \& Weill, L. (2015). Does Islamic banking development favor macroeconomic e $\varnothing$ ciency? Evidence on the Islamic finance-growth nexus. Economic Modelling, 47, 32-39. 
Hassan, M. K., \& Aliyu, S. (2018). A Contemporary Survey of Islamic Banking Literature. Journal of Financial Stability, 34, 12-43.

Hayashi F. (1982). Tobin's marginal Q and average Q: A neoclassical interpretation. Econometrica, 50, 213-224.

Ibrahim, M. H., \& Rizvi, S. A. R. (2017). Do We Need Bigger Islamic Banks? An Assessment of Bank Stability. Journal of Multinational Finance Management, 40, 77-91.

Ibrahim, M. H., \& Rizvi, S. A. R. (2018). Bank Lending, Deposits and Risk-Taking in Times of Crisis: A Panel Analysis of Islamic and Conventional Banks. Emerging Markets Review, 35, 31-47.

Imam, P., \& Kpodar, K. (2010). Islamic banking: How has it spread? International Monetary Fund, Working Paper.

Imam, P., \& Kpodar, K. (2016). Islamic banking: Good for growth? Economic Modelling, 59, 387-401.

Jawadi, F., Cheffou, A., \& Jawadi, N. (2016a). Can the Islamic bank be an emerging leader? A panel data causality analysis. Applied Economics Letters, 23(14), 991-994.

Jawadi, F., Cheffou, A. I., \& Jawadi, N. (2016b). Do Islamic and conventional banks really differ? A panel data statistical analysis. Open Economies Review, 27(2), 293-302.

Jawadi, F., Jawadi, N., Ben Ameur, H., \& Idi Cheffou, A. (2017). Does Islamic banking performance vary across regions? A new puzzle. Applied Economics Letters, 24(8), 567-570.

Jawadi, F., \& Sousa, R. M. (2014). The relationship between consumption and wealth: A quantile regression approach. Revue D'économie Politique, 124(4), 639-652.

Kabir, M. N., \& Worthington, A. C. (2017). The 'Competition-Stability/Fragility' Nexus: A Comparative Analysis of Islamic and Conventional Banks. International Review of Financial Analysis, 50, 111-128.

Kabir, M. N., Worthington, A., \& Gupta, R. (2015). Comparative Credit Risk in Islamic and Conventional Bank. Pacific-Basin Finance Journal, 34, 327-353.

Koenker, R. \& Xiao, Z. (2006) Quantile Autoregression. Journal of the American Statistical Association, 101(2), 980-1006.

Koenker, R. (2005). Quantile Regression. Econometric Society Monograph Series, Cambridge University Press, Cambridge.

Koenker, R., \& Hallock, K. F. (2001). Quantile regression. Journal of economic perspectives, 15(4), 143-156.

Koenker, R., \& Bassett, G.S. (1978). Regression quantiles. Econometrica, 46, 33-50. 
Lewis, M.K.L., \& Algaoud, L.M. (2001). Islamic Banking. Edward Elgar Publishing, Cheltenham, UK.

Lindenberg, E. B., \& Ross, S. A. (1981). Tobin's q ratio and industrial organization. Journal of Business, 1-32.

Narayan, P. K., \& Phan, D. H. B. (2019). A survey of Islamic banking and finance literature: issues, challenges and future directions. Pacific-Basin Finance Journal, 53, 484-496.

Olson, D., \& Zoubi, T. (2017). Convergence in Bank Performance for Commercial and Islamic Banks During and After the Global Financial Crisis. The Quarterly Review of Economics and Finance, 66, 71-87.

Skinner, D. J. (1993). The investment opportunity set and accounting procedure choice: Preliminary evidence. Journal of accounting and economics, 16(4), 407-445.

Yanikkaya, H., Gumus, N., \& Pabuccu, Y. U. (2018). How Profitability Differs Between Conventional and Islamic banks: A Dynamic Panel Data Approach. Pacific-Basin Finance Journal, 48, 99-111.

Zantioti, L. E. (2009). Does Islamic bank performance dier by region? $\mathrm{PhD}$ Thesis, Stockholm School of Economics.

Zins, A., \& Weill, L. (2017). Islamic Ranking and Risk: The Impact of Basel II. Economic Modelling, 64, 626-637. 